\title{
Treatment of Pelvic Organ Prolapse with "Vaginal Laser"- A Mini-Review of the Literature
}

\author{
Yaman Degirmenci*, Christine Skala and Annette Hasenburg \\ Department of Gynaecology and Obstetrics, University Medical Center Mainz, Mainz, Germany \\ *Corresponding author: Yaman Degirmenci, Department of Gynaecology and Obstetrics, University Medical Center \\ Mainz Langenbeckstr 1, 55131, Mainz, Germany
}

\section{ARTICLE INFO}

Received: 幽 July 24, 2021

Published: 幽 August 04, 2021

Citation: Yaman Degirmenci, Christine Skala and Annette Hasenburg. Treatment of Pelvic Organ Prolapse with "Vaginal Laser"- A Mini-Review of the Literature. Biomed J Sci \& Tech Res 37(5)-2021. BJSTR. MS.ID.006059.

\begin{abstract}
The pelvic organ prolapse (POP) is a common condition of women at any age and affects the quality of life negatively. The technology that is known as "laser treatment" has been used more often recently as a part of studies to treat POP. In this paper we reviewed the databases (PubMed, Google Scholar, and Web of Science) for published literature about the use of laser treatment of POP in women and identified relevantpapers.
\end{abstract}

Keywords: Pelvic Organ Prolapse; POP; Vaginal Laser; Er: YAG Laser; Microablative Fractional $\mathrm{CO}_{2}$ Laser

\section{Introduction}

It is not yet possible to prevent the aging processes. Like other several risk factors (such as genetics, race/ethnicity, gender, obstetrical and gynaecological injuries, smoking, obesity) the aging also causes weakening of the pelvic floor connective tissue/ collagen and leads to pelvic organ prolapse (POP) [1]. Although the exact prevalence of the pelvic organ prolapse of women is unknown, this condition was reported up to 50 percent in some studies [1]. Most women having a POP, often complain of a pressure sensation respectively a bearing-down sensation [2]. The POP impairs further, as a consequence of its impact on bladder, bowel function and the sexual function, hence impacts the quality of life negatively [3]. The prolapse of pelvic organs can be treated either conservatively or surgically, each with its own advantages and disadvantages. Treatment with pessaries and the initiation of pelvic floor physical therapy with or without estrogens are the usual effective conservative treatment options for POP of women $[4,5]$. Surgical treatment is the other alternative for qualified cases. Surgery requires experience and can require more effort and it may cause more complications. The laser treatment is getting increasingly popular in the field of urogynaecology as an alternative option, following its adoption from dermatology and plastic-surgery. The motto of this procedure is the reversal of aging „rejuvenation“. In this paper we reviewed the literature for the use of laser for POP in women.

\section{Vaginal Laser Technology}

The laser light energy induces a controlled injury by means of thermal or ablative effect at the superficial tissue level $[6,7]$. Microablative fractional CO2 and Er: YAG (erbium-doped yttrium aluminium garnet) lasers are two introduced systems to treat genitourinary conditions. Two technologies differ from each other in terms of their acting principals and varying wavelengths. The absorbed waves in the tissue leads to an expected tissue reaction. Microablative fractional CO2 laser creates superficial microablation zones on the mucosal surface. In contrast to CO2 laser creates Er:YAG laser rapid sequential heat pulses, which are transferred into the mucosa. The fast heat pulse does not injure the tissue surface [8]. Hypothesized as "tissue remodelling" showed by Gaspar, et al. in 2011 [9-11] showed in 2015 that the laser treatment induces neocollagenesis, fibrillogenesis and neovascularisation. 


\section{Laser in Gynaecology/Urogynaecology}

The laser technology has been widely used in gynaecology for more than 40 years after Holmquist et al. first introduced the use of laser in vaginal surgery in the 1970s [12]. US Food and Drug Administration (FDA) licenced the laser systems for "incision, excision, ablation, vaporization and coagulation of body soft tissues [7]. Other than this indications, laser is increasingly being used for the treatment of vaginal atrophy, urinary incontinence, vulvodynia, lichen sclerosus and vaginal laxity syndrome. However but there's still lacking good-quality evidence and long term follow-up studies [13-15].

\section{Laser Treatment for Pelvic Organ Prolapse of Women}

Collagen maintains the supportive functions of the pelvic floor and is one of the most important components of the extracellular matrix in connective tissue. Jackson et al. presented a hypothesis to explain the mechanism for genitourinary prolapse on a molecular level. They showed that genitourinary prolapse is associated with a reduction in total collagen content and a decrease in collagen solubility [16]. The Hypothesis is that the prolapse would be improved after a laser treatment was related to the reality of „tissue remodelling" with laser injury. Just a few studies in the literature discussed the laser treatment for POP as a primary endpoint. Ogrinc et al. published a pilot study of 61 patients with grade II-IV cystocele classified using the Baden-Walker halfway system. Patients received between two and five Er:YAG laser treatment sessions at 2-month intervals. They showed significant improvement even after the first treatment session in prolapse stages and 95\% of cystoceles improved by at least one grade without major adverse effects. Follow up of this study was 12 months [17]. Another publication by the same group was able to show that the improvement lasted at least 12 months and for many patients even longer up to 36 months without significant treatment discomfort (average score of 0.4 on a 10-point VAS) and with high patient satisfaction (median level of 4 on a scale of 1-5) [18]. The authors reported no adverse effects on that one as well. Sipos et al. enrolled 40 postmenopausal women with genitourinary symptoms of menopause (GSM) into a prospective cohort study. The treatment was carried out with a microablative $\mathrm{CO} 2$ laser in 3 sessions with 4-6 weeks apart. The authors showed an statistically significant improvement in pelvic organ prolapse distress with a questionnaire (Pelvic Organ Prolapse Distress Inventory- POPDI-6) after the second and third treatment (POPDI-6 (mean \pm SD) baseline $21 \pm 18$ / after first treatment $17 \pm$ 15 / after second treatment $14 \pm 15$ / after third treatment $13 \pm 13$ p-value $0.44 / 0.03 * / 0.01 *$ ) [19].

Gaviria et al. evaluated in a pilot study of 21 patients who received 2 Er:Yag laser treatment sessions for vaginal tightening. 5 patients $(23.8 \%)$ showed by the first measurements previous to the treatment prolapses (assessment with POP-Q classification) of stages 1 to 3 . The authors defined an improvement in prolapse after laser treatment (four patients improved by one stage and one showed improvement of two POP-Q stages). The type of prolapse in this study was not clearly defined [20]. Athanasiou et al. showed in a current study that no improvement for POP in a randomised, single-blind study with Er:YAG laser treatment [21]. The authors compared watchful waiting in thirty (15 vs. 15) postmenopausal women having a cystocele and/or rectocele stage 2 or 3 with applied 3 Er:YAG laser treatments at monthly intervals. In one Study showed Lin et al. also as a secondary end point the improvement of POP symptoms assessed with questionnaires after vaginal laser treatment for other indications [22].

\section{Discussion}

This review covers mainly just 6 publications to interpret the effect of laser treatment on POP in women. Both Er:YAG and microablative fractional $\mathrm{CO} 2$ were utilized for treatment. One Study commented the effect for pelvic organ prolapse as a secondary outcome and just one of the studies had a control group. The rest were observational studies. None of the studies compared the laser with a usual effective conservative (PFPT-Pessar) treatment method [4,5]. Ogrinc et al. applied multiple passes of laser irradiation in a treatment session depending on the prolapse severity to treat POP without a clearly defined assignment which leads to a protocol weakness [17]. Sipos AG et al. excluded the patients with >Stage II POP (according to the pelvic organ prolapse quantification system POP-Q) and ask the participants to complete the Pelvic Floor Distress Inventory (PFDI-20) questionnaire, which has three components: Pelvic Organ Prolapse Distress Inventory 6 (POPDI-6), Colorectal-Anal Distress Inventory 8 (CRADI-8), and Urinary Distress Inventory (UDI-6). The improvement by POP after laser treatment could only be shown regarding the POPDI-6 Questionnaire and not supported with a clinical examination or re-classification of POP according to POP-Q [19]. Gaviria et al. used the PISQ-12 questionnaire to evaluate the improvement after laser treatment [20]. These combined questionnaires in means of sexuality, incontinence and prolapse make it harder to interpret the results based on POP. The lack of a clear definition of the type of POP and the low number of patients with POP makes this study interpretation in terms of POP laser treatment weaker. In our opinion the improvement of scores for questionnaires should also be supported with clinical stratification to obtain significant results. The longest follow up was limited to 36 months [17]. That frustrates also us to speak about long- term effects of laser. The two discrepant studies $[18,21]$ also show us that the age of the patient, observing time and treatment frequency matters. The treatment modalities should be that's why improved/individualized. Ogrinc et al. commented also on this study and attributed the discrepancy to the study design [23]. No major adverse effect is reported 
eventually for laser treatment by POP. A large observational 8 years follow up study which aimed to show the safety of Er:YAG laser may also supports this finding [24].

\section{Conclusion}

As seen above although the literature is hopeful there's still lacking data on the safety and the effectiveness of laser treatment in POP. That's why issued FDA in 2018 states a warning concerning effectiveness and safety of laser treatment for urinary incontinence, vaginal "rejuvenation" or cosmetic vaginal procedures [7]. The published studies are leading and lightening the scientific way. That should motivate the clinicians to design better planned randomised and controlled prospective studies to understand the effect of the laser treatment by POP in woman. We believe that laser treatment will soon be used and recommended as an alternative, minimally invasive treatment method for low grade POP.

\section{Conflict of Interest}

There are no conflicts of interest with regard to this publication.

\section{Funding}

No funding.

\section{References}

1. Weintraub AY, Glinter H, Marcus-Braun N (2020) Narrative review of the epidemiology, diagnosis and pathophysiology of pelvic organ prolapse. Int Braz J Urol 46(1): 5-14.

2. Chung SH, Kim WB (2018) Various Approaches and Treatments for Pelvic Organ Prolapse in Women. J Menopausal Med 24(3): 155-162.

3. Lamers BH, Broekman BM, Milani AL (2011) Pessary treatment for pelvic organ prolapse and health-related quality of life: a review. Int Urogynecol J 22(6): 637-644.

4. Li C, Gong Y, Wang B (2016) The efficacy of pelvic floor muscle training for pelvic organ prolapse: a systematic review and meta-analysis. Int Urogynecol J 27(7): 981-992.

5. Griebling TL (2016) Vaginal pessaries for treatment of pelvic organ prolapse in elderly women. Curr Opin Urol 26(2): 201-206.

6. Ratz C (2019) Vaginal laser therapy for urinary incontinence and genitourinary syndrome of menopause: A review. Urologe A 58(3): 284290.

7. Preti M, Vieira-Baptista P, Digesu GA, Bretschneider CE, Damaser M, et al. (2019) The clinical role of LASER for vulvar and vaginal treatments in gynecology and female urology: An ICS/ISSVD best practice consensus document. Neurourol Urodyn 38(3): 1009-1023.

8. Mitsuyuki M, Stok U, Hreljac I, Yoda K, Vizintin Z (2020) Treating Vaginal Laxity Using Nonablative Er:YAG Laser: A Retrospective Case Series of Patients From 2.5 Years of Clinical Practice. Sex Med 8(2): 265-273.
9. Gaspar A AG, Brandi H (2011) Vaginal Fractional CO 2 Laser: A Minimally Invasive Option for Vaginal Rejuvenation. Am J Cosmet Surg 28(3): 156162.

10. Salvatore S, Leone Roberti Maggiore U, Athanasiou S, Origoni M, Candiani $\mathrm{M}$, et al. (2015) Histological study on the effects of microablative fractional $\mathrm{CO} 2$ laser on atrophic vaginal tissue: an ex vivo study. Menopause 22(8): 845-849.

11.Zerbinati N, Serati M, Origoni M, Candiani M, Iannitti T, et al. (2015) Microscopic and ultrastructural modifications of postmenopausal atrophic vaginal mucosa after fractional carbon dioxide laser treatment. Lasers Med Sci 30(1): 429-436.

12. Holmquist ND, Bellina JH, Danos ML (1976) Vaginal and cervical cytologic changes following laser treatment. Acta Cytol 20(4): 290-294.

13. Bhide AA, Khullar V, Swift S, Digesu GA (2019) The use of laser in urogynaecology. Int Urogynecol J 30(5): 683-692.

14. Mackova K, Van Daele L, Page AS, Geraerts I, Krofta L, et al. (2020) Laser therapy for urinary incontinence and pelvic organ prolapse: a systematic review. BJOG 127(11): 1338-1346.

15. Franic D, Fistonic I (2019) Laser Therapy in the Treatment of Female Urinary Incontinence and Genitourinary Syndrome of Menopause: An Update. Biomed Res Int 2019: 1576359.

16. Jackson SR, Avery NC, Tarlton JF, Eckford SD, Abrams P, et al. (1996) Changes in metabolism of collagen in genitourinary prolapse. Lancet 347(9016): 1658-1661.

17. Ogrinc UB, Sencar S (2017) Non-ablative vaginal erbium YAG laser for the treatment of cystocele. Ital J Gynaecol Obstet 29(1): 19-25.

18. Ogrinc UB, Sencar S, Vizintin Z (2017) \#178 3 years follow-up of pelvic organ prolapses treated with Er:YAG laser. Lasers Surg Med 49(S28): 63.

19. Sipos AG, Kozma B, Poka R, Larson K, Takacs P (2019) The Effect of Fractional CO2 Laser Treatment on the Symptoms of Pelvic Floor Dysfunctions: Pelvic Floor Distress Inventory-20 Questionnaire. Lasers Surg Med 51(10): 882-886.

20. Gaviria J, Lanz J (2012) Laser Vaginal Tightening (LVT) - evaluation of a novel noninvasive laser treatment for vaginal relaxation syndrome. Laser Heal Acad 2012(1): 59-66.

21. Athanasiou S, Pitsouni E, Cardozo L, Zacharakis D, Petrakis E, et al. (2021) Can pelvic organ prolapse in postmenopausal women be treated with laser therapy? Climacteric 24(1): 101-106.

22. Lin KL, Chou SH, Long CY (2019) Effect of Er:YAG Laser for Women with Stress Urinary Incontinence. Biomed Res Int 2019: 7915813.

23. Ogrinc UB, Sencar S (2021) Comment: 'Can pelvic organ prolapse in postmenopausal women be treated with laser therapy?'. Climacteric 24(2): 210.

24. Gambacciani M, Cervigni M, Gaspar A, Novakov Mikic A, Gaviria J, et al. (2020) Safety of vaginal erbium laser: A review of 113,000 patients treated in the past 8 years. Climacteric 23(1): S28-S32. 
ISSN: 2574-1241

DOI: 10.26717/BJSTR.2021.37.006059

Yaman Degirmenci. Biomed J Sci \& Tech Res

cC (P) This work is licensed under Creative

Submission Link: https://biomedres.us/submit-manuscript.php

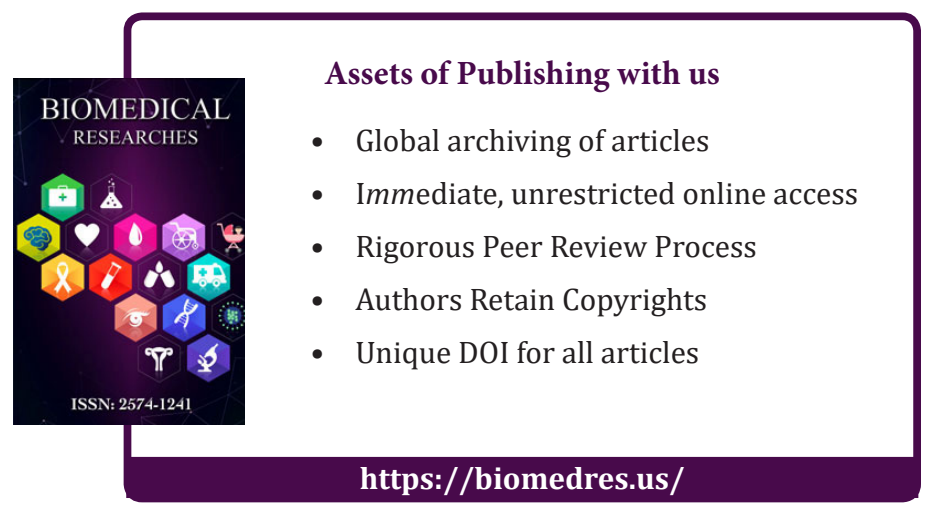

